\title{
ROBOTIC APLICATIONS IN SURGERY WITH SPECIAL EMPHASIS ON APLICATIONS IN NEUROSURGERY
}

\author{
NikOLIC, G.; JeRBIC, B. \& CHUdy, D.
}

\begin{abstract}
This paper gives a description of historical development and current achievements in the field of the application of robots in surgery with special emphasis on robotic neurosurgery. Some examples of the application of robots in surgery and particularly in neurosurgery are described. Advantages and disadvantages of the described solutions of neurosurgical robots are presented. Since the authors are developing their own neurosurgical robot named RONNA, a more detailed description of its configuration and possible advantages compared to other existing solutions is provided. A principle explanation of the operating principle of the system is given. Specific solutions for the localization of the patient as well as connecting the robot system with tomography images (CT or MRI) are described. The developed modules of the RONNA system, which are interconnected and include planning software, measuring devices, navigation system, stereovision calibration, surgical instruments and advanced software, are presented. The operating principle of each module is described, and the solutions performed are presented. The RONNA system is under preclinical studies which are expected to provide further guidelines for the development and harmonization with the environment of the operating room. The authors' viewpoint of the future development of robots in surgery and particularly in neurosurgery is given. Present trends in the development of micro and nano robots in medicine, designed for performing medical procedures within the patient's body without surgical intervention from the outside are also mentioned.
\end{abstract}

Key words: neurosurgery, neurosurgical robots, neurosurgical navigation, neurosurgical robot RONNA, stereotaxis
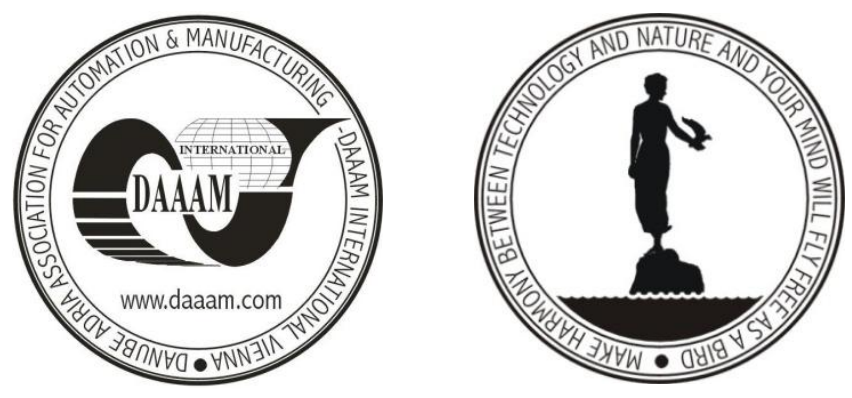

Authors' data: Nikolic, G[ojko]*; Jerbic, B[ojan]**; Chudy, D[arko]*** *University of Zagreb, Faculty of Textile Technology, ** University of Zagreb, Faculty of Mechanical Engineering and Naval Architecture, *** Clinical Hospital Dubrava, Zagreb, gojko.nikolic@ttf.hr, bojan.jerbic@fsb.hr,

This Publication has to be referred as Nikolic, G[ojko]; Jerbic, B[ojan] \& Chudy, D[arko] (2013) Robotic Applications in Surgery with Special Emphasis on Applications in Neurosurgery, Chapter 06 in DAAAM International Scientific Book 2013, pp. 149-172, B. Katalinic \& Z. Tekic (Eds.), Published by DAAAM International, ISBN 978-3-901509-94-0, ISSN 1726-9687, Vienna, Austria

DOI: $10.2507 /$ daaam.scibook.2013.06 
Nikolic, G.; Jerbic, B. \& Chudy, D.: Robotic Applications in Surgery with Special...

\section{Introduction}

The term engineering often implies only mechanical engineering aspects of the application in industry. However, this term increasingly goes beyond the usual limits and appears in all other forms of human activity; it can be generally seen as the application of integrated and polydisciplinary engineering processes in order to achieve better, faster and higher quality results. In this context robotics should be considered as a particularly relevant engineering area, especially its application in medicine. Medicine is today unthinkable without the use of sophisticated technical devices.

The application of robotics in medicine has been present for only twenty years, but its impact is increasing, primarily on surgical procedures. There is almost no area of medicine where robots have not been applied in some form, including ophthalmology, urology, gynaecology, cardiology, neurology, orthopaedics, etc. Surgical robotics is the most fascinating interdisciplinary field of medical engineering. Neurosurgery is especially suitable for the application of robots, and in that sense there are more advanced solutions, but most of them are not applied. The reasons should be sought primarily in people's attitudes towards robots. Even first applications of robotic technology for medical purposes, especially in neurosurgery, provoked many ethical issues as no other device used in medicine.

The first one is visual "similarity" with man, and the second one relates to the possible "independence" of robots in decision making. In reality, every complex medical device that is used directly or indirectly in a patient's treatment may suffer from a defect and cause consequences for the patient. Despite this primary scepticism advantages offered by the application of robots are significant. Due to the development of intelligent management software and sensor systems, the robot can replace the surgeon in his functions and such a replacement may be significantly "more effective". This contributes to the quality of interventions, reducing the traumatic consequences for the patient and accelerates his convalescence.

Possible application of robots in medicine is wide and not limited on surgery but also in laboratories, operation and care of patients, assisting doctors in diagnostics, etc. Robotics in medicine develops in many different directions: assisting in performing surgery, independent performance of precise surgery and activities within the patient's body using miniature robots. New robotic applications require new solutions, both in terms of structure and management methods, and in the application of new materials and sensors. Combinations of organic and inorganic materials to create bionic robots with the trend of "using" the robot made entirely "of living matter," or genetically modified organisms that perform programmed functions are used in experiments. Therefore, the concept of robotics is gradually changing in relation to the established and existing definitions derived from the earlier use of robots exclusively for industrial purposes. 


\section{Features of Previous Surgical Robots}

\subsection{Definitions}

Past applications of the robot in industry have determined its definition. The desire to connect the term robot exclusively with one precisely defined form of a complex device has created the definitions whose large number is targeted at industrial robots, e.g.: RIA - Robot Institute of America, JIRA - Japan Industrial Robot Association; ISO - International Organization for Standardization, as changed definitions in some countries such as Germany and England, which added their amendments (Nikolic et al., 2008). Regardless of the ultimate definition the industrial robot was exclusively kept in mind. Only one recent definition that reads "robot is a modern multi-purpose automated machine that replaces the physical and (increasingly) the rational work of man" (Prof. B. Novaković, Faculty of Mechanical Engineering and Naval Architecture, Zagreb, Croatia), gives a broader definition outside the exclusive use in industry. However, even in this definition the new trends in the development of miniature robots cannot fit in.

Along with new applications new definitions are given, e.g. (Herron \& Marohn, 2007). "Robotic surgery" is defined as "a surgical procedure or technology that uses sophisticated computer devices in the interaction between a surgeon and a patient during a surgical operation and assumes some degree of control heretofore completely reserved for the surgeon."

It is logical that the definitions always try to show a "pre-existing" condition and can hardly consider an unpredictable development and future application versions. Therefore, although it is not a characteristic of science, definitions should be left aside for the time being, because the rapid development of robotics in the broadest sense makes every definition limited to a particular application and related to the time of origin.

In surgery the term Computer-Integrated Surgery - CIS, which is to some extent a predecessor of the application of surgical robots and indicates areas of intervention technologies in medicine with the help of image processing - "navigation" (CT or MRI), is used. CIS also includes surgical CAD / CAM (Computer Aided Design and Manufacturing), which is analogous to industrial CAD / CAM, with the difference that this digital information is used to create mathematical models for making surgical plans or improving therapy (Haidegger 2, 2008). This technique is already a standard procedure in medicine and is considered to have been used for hundreds of thousands of surgical operations.

The application of robotics in medicine began a little more than twenty years ago. However, nowadays it is a rapid and unstoppable trend that in the near future it will replace the classic practice of interventional medicine (Advincula et al., 2007). The application of robots in surgery originated with the aim to overcome limitations in existing surgical procedures with minimally invasive surgery. Robotics in surgery is one of the most complex and most sophisticated interdisciplinary engineering fields of work. Within surgery the neurosurgery is one of the most challenging fields because the complexity of anatomical structure, high sensitivity and delicate functionality of tissue require extremely precise faultless procedures. The application 
of robots in surgery is often referred to as robotic surgery or computer-assisted surgery (Anonymous 9, 2013). Robots may be involved in medical procedures in different ways and with varying degrees of independence or interaction with a surgeon and a patient. One of them plays the role of dependent "assistant", i.e. a surgical operation is controlled by the surgeon as shown in Figure 1-a. Advantages are immense from avoiding tremor (due to subtle shifts or due to fatigue), achieving the accurate depth of the penetration of tools as well as the exact way of spatial movement. Examples of such a procedure are biopsy, placement of electrodes and catheters, bone drilling etc. Robots that perform these operations independently without the "leadership" of the surgeon are autonomous robots (Figure 1-b), and although all technical issues have been generally solved, they are not widely used primarily for ethical reasons outlined in the introduction.

Teleoperating devices or devices with remote control (like laparoscopic robotic da Vinci and Zeus System), Figure 1-c, are the third robot type (though no robots by any definition or characteristics, but so called in the available literature). These are the devices that have three key components: actuating manipulators ( 3 to 5), usually laparoscopic instruments, then the main control device or system, and vision system with a console for the operator. The procedure comes down to the remote control of actuating manipulators (laparoscopic instruments) based on finger movements and foot pedals of a trained surgeon who observes the condition of the patient's body on the enlarged screen (up to 10x) and controls medical instruments. This distance does not need to be just a few meters inside the operating room, but can be longer distances between cities and regions. The military is especially interested in this method of operation because of possible surgical operations directly on the battlefield by a remote surgical team.

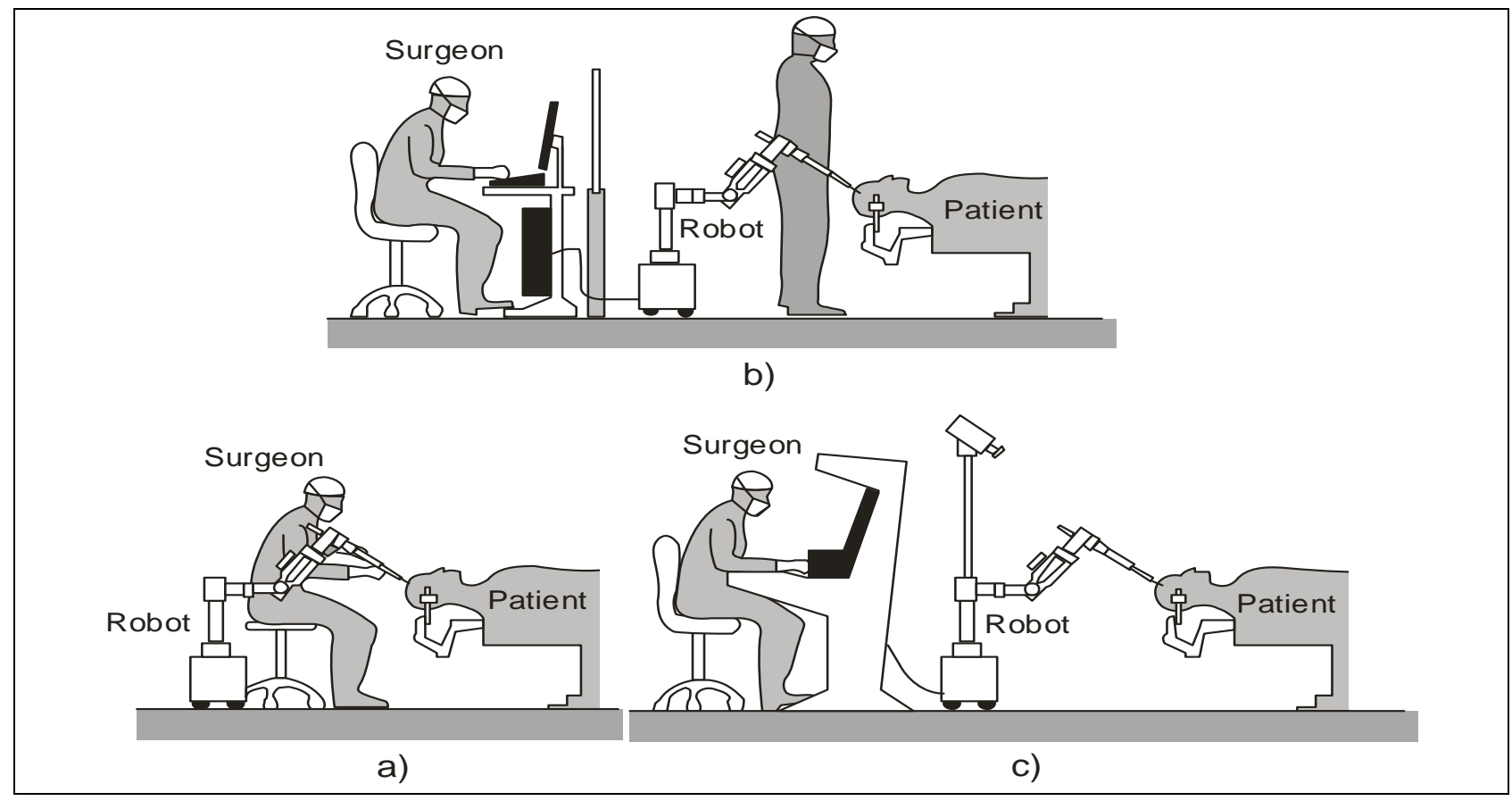

Fig. 1. Ways of using robotic neurosurgery: a) robot slave guiding a robot arm, b) managing the independent robot, c) teleoperated remote control of robot manipulators (drawn according to Anonymous 10, 2005) 


\subsection{History of the Application of Robots in the Area of Surgery and Neurosurgery}

Today, one can say that robotic surgery has firmly taken its place in medicine thanks to interdisciplinary teams of engineers and doctors. According to Medical Robotic Database - MeRoDe, there are more than 200 research projects dedicated to the application of robots in surgery (Haidegger 1, 2008). Regardless of success, most of the projects have not been commercialized for various reasons.

The first application of robots in surgery was for neurosurgery in 1985 when the industrial robot PUMA 560 was used to place a needle for a brain biopsy using CT guidance (Karli \& Ćustić 2007), Figure 2. It is not unusual that robots were first used in neurosurgery. The reason for this is that the brain compared to the captured image of CT retains the same position very accurately and the robot can precisely reach the target point.

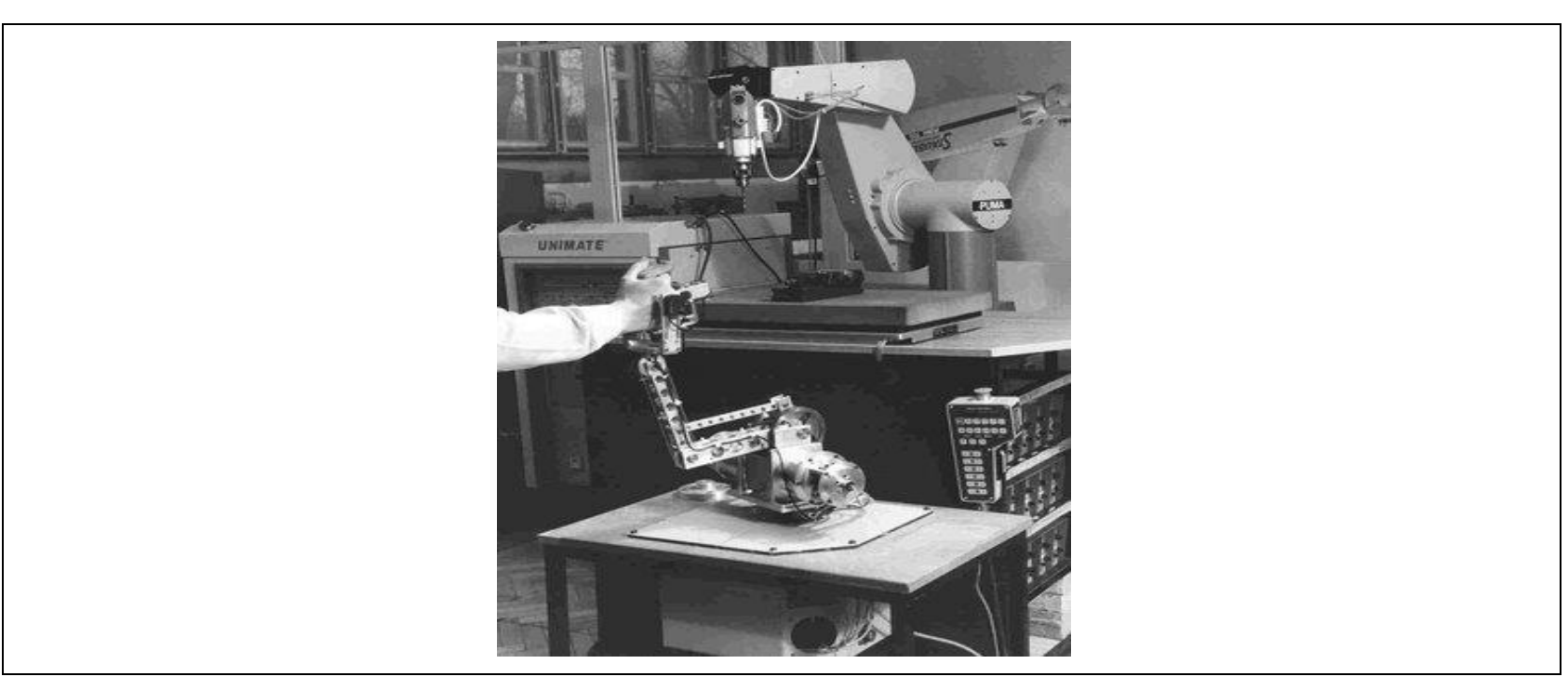

Fig. 2. Puma 560 robot (Anonymous 8, 2012)

In 1988, the PROBOT was developed at Imperial College London and was used to perform prostatic surgery (Anonymous 9, 2013).

The AESOP robotic surgical system was introduced in 1990 and was the first system to be approved by FDA (Food and Drug Administration) for endoscopic surgical procedures.

The Company Integrated Surgical Systems developed the ROBODOC) which was introduced in 1992 to integrate precise fittings in the femur for hip replacement. It was the first surgical robot in orthopaedic surgery approved by FDA (Anonymous $2,2004)$. One of the mostly used robotic systems is the telesurgery system da Vinci, Fig. 3.

The prototype was made in 1997 and in 1998 the first heart bypass surgery was performed. In USA its application was approved by FDA in 2000 and in Europe it has been used since 2001. It was developed by SRI International in Menlo Park supported immensely by the agencies DARPA (Defense Advanced Research Projects Agency) and NASA (National Aeronautics and Space Administration). Computer Motion with AESOP developed the similar telesurgery system ZEUS firstly used to 
Nikolic, G.; Jerbic, B. \& Chudy, D.: Robotic Applications in Surgery with Special...

operate fallopian tube in Cleveland in 1997. Intuitive Surgical bought patent rights for Da Vinci and acquired Computer Motion in 2003. This is the reason why ZEUS is not sold any more (Anonymous 2, 2004), possibly for commercial reasons so that only one system Da Vinci remains on the market.

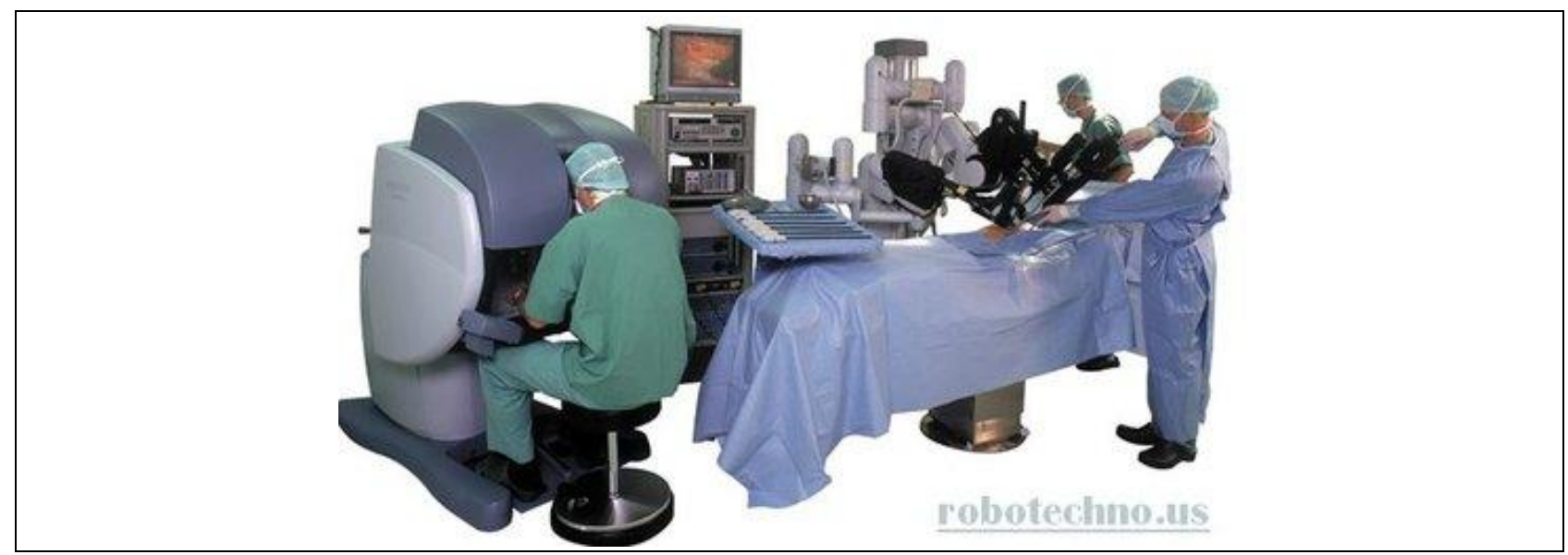

Fig. 3. Teleoperating robot Da Vinci (Anonymous 8, 2012)

Da Vinci was developed as a surgical robot for remote surgery and was originally designed to perform surgical procedures on distant battlefields and in other hazardous environments, but finally it has turned out that it has a higher utility value for minimally invasive surgery in the operating room (Anonymous 8, 2012). Its application is enormous, and there are more than 2,000 units in hospitals worldwide (January 2013); more than 200,000 surgeries were performed using this surgical robot in one year (data for 2012) (Anon. 1, 2013). The first surgery was performed at the Ohio State University Medical Center in Columbus, Ohio (Anonymous 9, 2013).

It is used for a variety of surgical procedures in gynaecology, urology, abdominal surgery, orthopaedic surgery, spine surgery, cardiology, etc. Recently it has been used for organ transplantation.

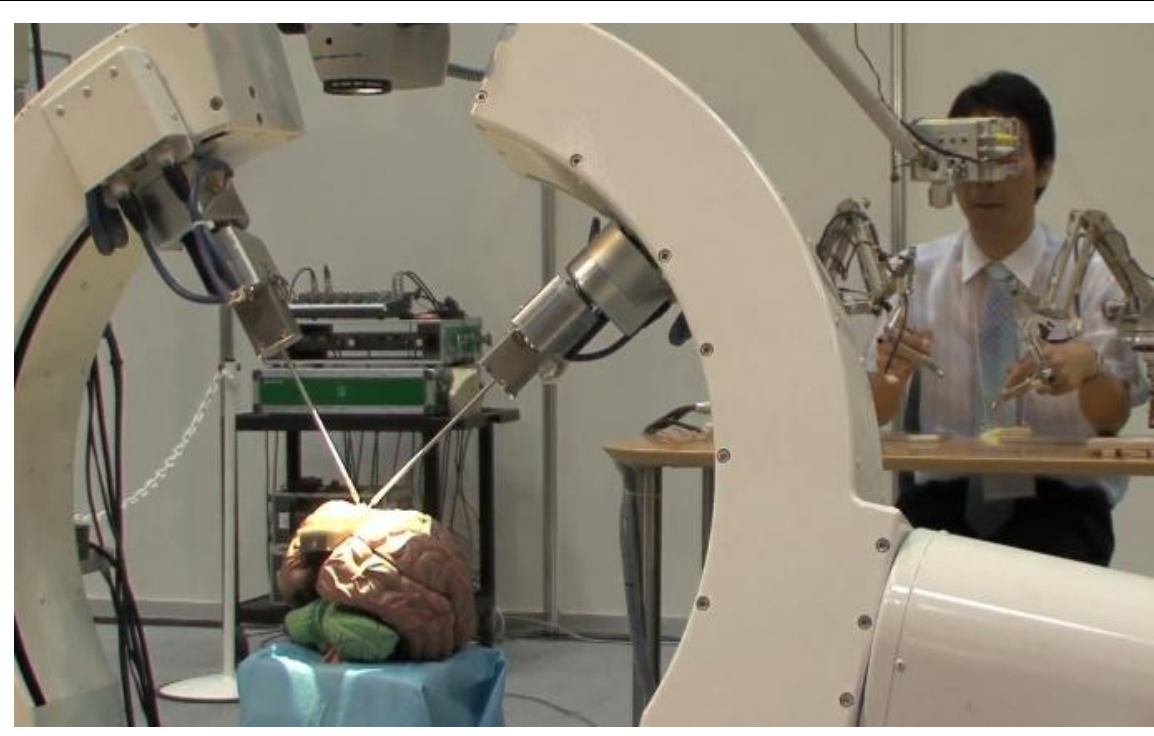

Fig. 4. Japanese micro-surgery support system for neurosurgery

(Anonymous 5, 2009) 
Mitsuishi-Sugita University of Tokyo, developed the teleoperating "Microsurgery support system for neurosurgery" in 2010, Figure 4. The system is designed for reconnecting blocked blood vessels near the surface of the brain. Coronary bypass surgery requires high precision, which is also demanding for an experienced surgeon. An important advantage of this system is exceptional accuracy, which is achieved, according to the data presented, with a controlled translation of the robot of only 10 microns. Monitoring is done by 3D cameras that show the area of surgery on a 3D monitor. The time lag is around $100 \mathrm{~ms}$, which is satisfactory for movement coordination (management). Actuating manipulators with surgical instruments have three degrees of freedom for translation, three for attitude and one for gripping. Precision and controlled movements without tremor, which can hardly be avoided because of free movements of the surgeon's hand, are essential. It is mentioned that in experiments it was possible to suture an artificial blood vessel $0.25 \mathrm{~mm}$ wide in five minutes. The system is not yet commercially available (Anon. 5, 2009).

We should also mention some of the robotic systems that are not significantly present in practice, such as:

- Alpha robot, a teleoperating system, developed by MicroDexterity Systems Inc:, Albuquerque, NM, USA, with 5 degrees of freedom (SS).

- KineMedic, a teleoperating system, developed by DLR/BrainLAB AG; Feldkirchen, Germany. A Light-Weight Robot (LWR) has a high capacity with 7 degrees of freedom. It is designed for minimally invasive surgery (MIS) in neurosurgery.

- MARS robot (SmartAssist) developed by Mazor Surgical Technologies Inc., Caesarea, Israel, the construction of which is made of a light-weight robot. It was approved by FDA and is commercially available.

Among major robotic systems are two robotic systems that are used in neurosurgery and are available on the market. One of them is NeuroMate, developed in France by IMMI / ISS / Schaerer Mayfield NeuroMate Sarl, from Lyon, which has 5 degrees of freedom and is designed for a wide range of functional neurosurgical procedures such as deep brain stimulation (DBS) and stereotactic electroencephalography (SEEG). Other applications require the use of stereotactic frames for neuroendoscopy, radiosurgery, biopsy, magnetic stimulation, etc., Figure 5. The robot can be used with a stereotactic frame or in frameless mode. When it is used with a stereotactic frame, there is no need to register as NeuroMate has already been calibrated within the coordinate system of the frame. When using frameless mode, localization is based on a special form of the localizing device. NeuroMate uses infrared sensors and ultrasound as a method to capture the common points required for registration. 
Nikolic, G.; Jerbic, B. \& Chudy, D.: Robotic Applications in Surgery with Special...

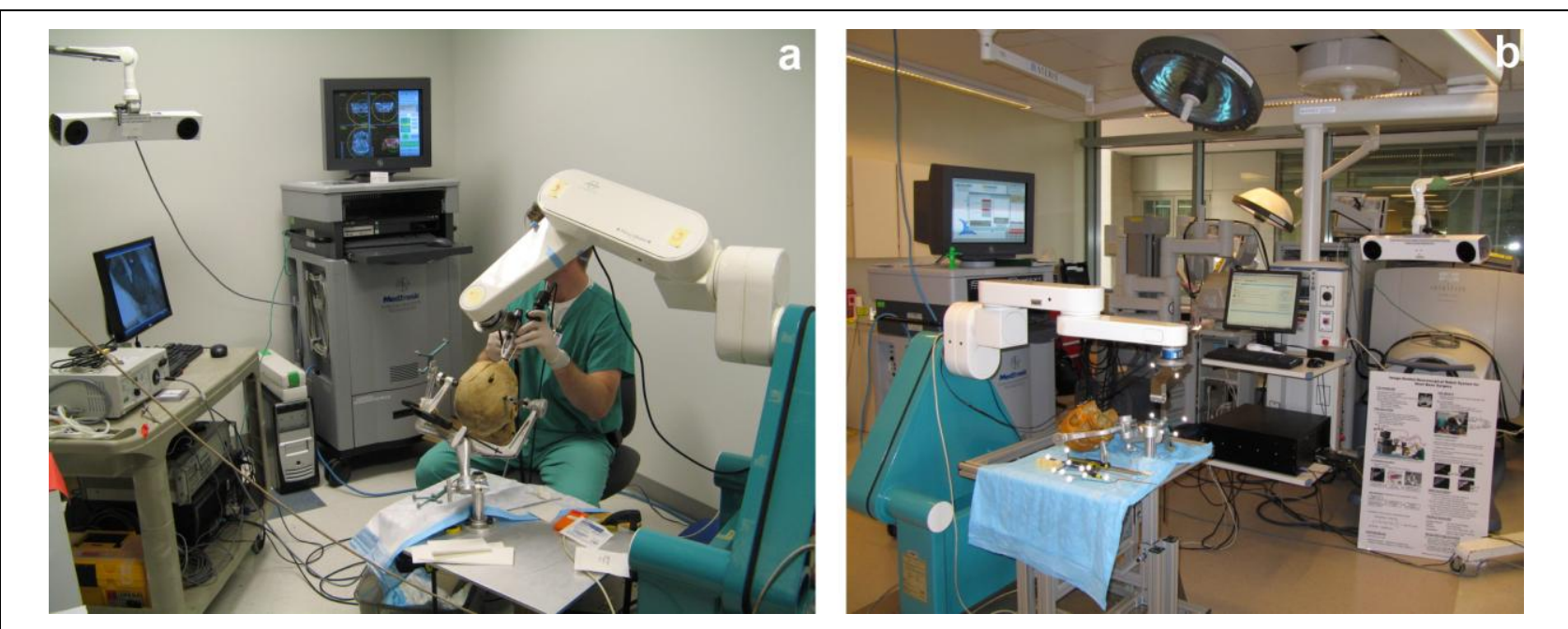

Fig. 5. NeuroMate neurosurgical robot in the laboratory (Haidegger 2, 2008)

Another robotic system used in neurosurgery with high expectations in the future is ROSA, designed by Medtech, Figure 6. It was developed in close collaboration with several surgical teams, using advanced robotic functions, navigation and haptic technology and advanced visualization capabilities. The localization of the ROSA robotic system is based on markerless registration. A neurosurgical planning software is integrated. The ROSA robotic device was approved for neurosurgical procedures by ISO 13485 and ISO 9001, obtained product conformity for its operation in Europe (CE) and it was also approved by Canadian Health Authorities and by FDA of USA (Anonymous 3, 2013).

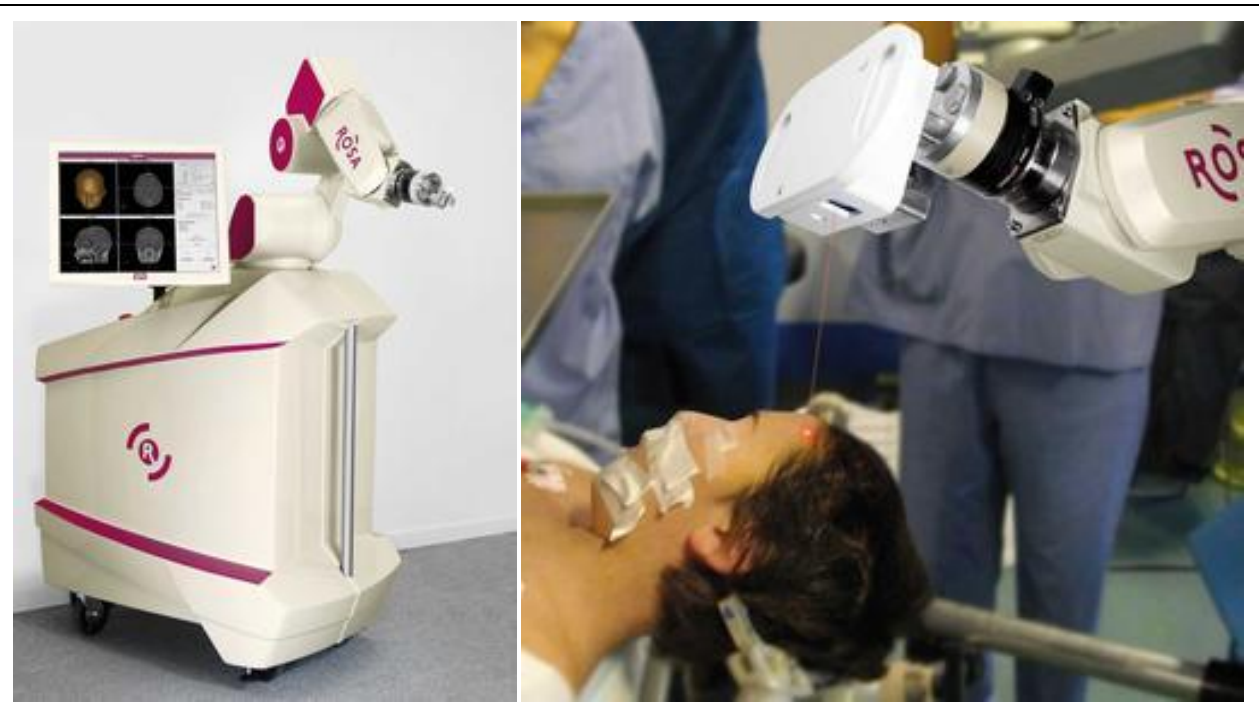

Fig. 6. ROSA neurosurgical robotic system, a) ROSA robot, b) ROSA robot in the operating room

One of the newest projects ROBOCAST for neurosurgery which is funded by EU united scientists from Germany, Italy, Israel and Great Britain on the mutual research work. In the framework of this project a new type of neurosurgical robot was developed that has significantly greater mobility in the operational area than the existing ones. A sensor system provides feedbacks to surgeons during surgeries that 
allow them to realistically evaluate the status of the surgical procedure, which allows for better and easier surgery. It successfully performed precise neurosurgical procedures in simulated surgeries.

Commercially available is also the portable neurosurgical robotic system Prosurgics Pathfinder designed by Prosurgics Ltd. High Wycombe, England, with 6 degrees of freedom, with a very stable base which can wheeled in and out of the operating room. The Pathfinder (Fig. 7) finds the surgical field using a reflector attached to the patient's head and a camera system integrated in the arm. Precise markers are impregnated with an opaque material visible in the CT and coated with a reflective material so that they can be registered with the camera. The markers are fixed to the skin with adhesive tape, or alternatively attached to the plate fixed to the skull. It is designed as a manipulator for guiding instruments.

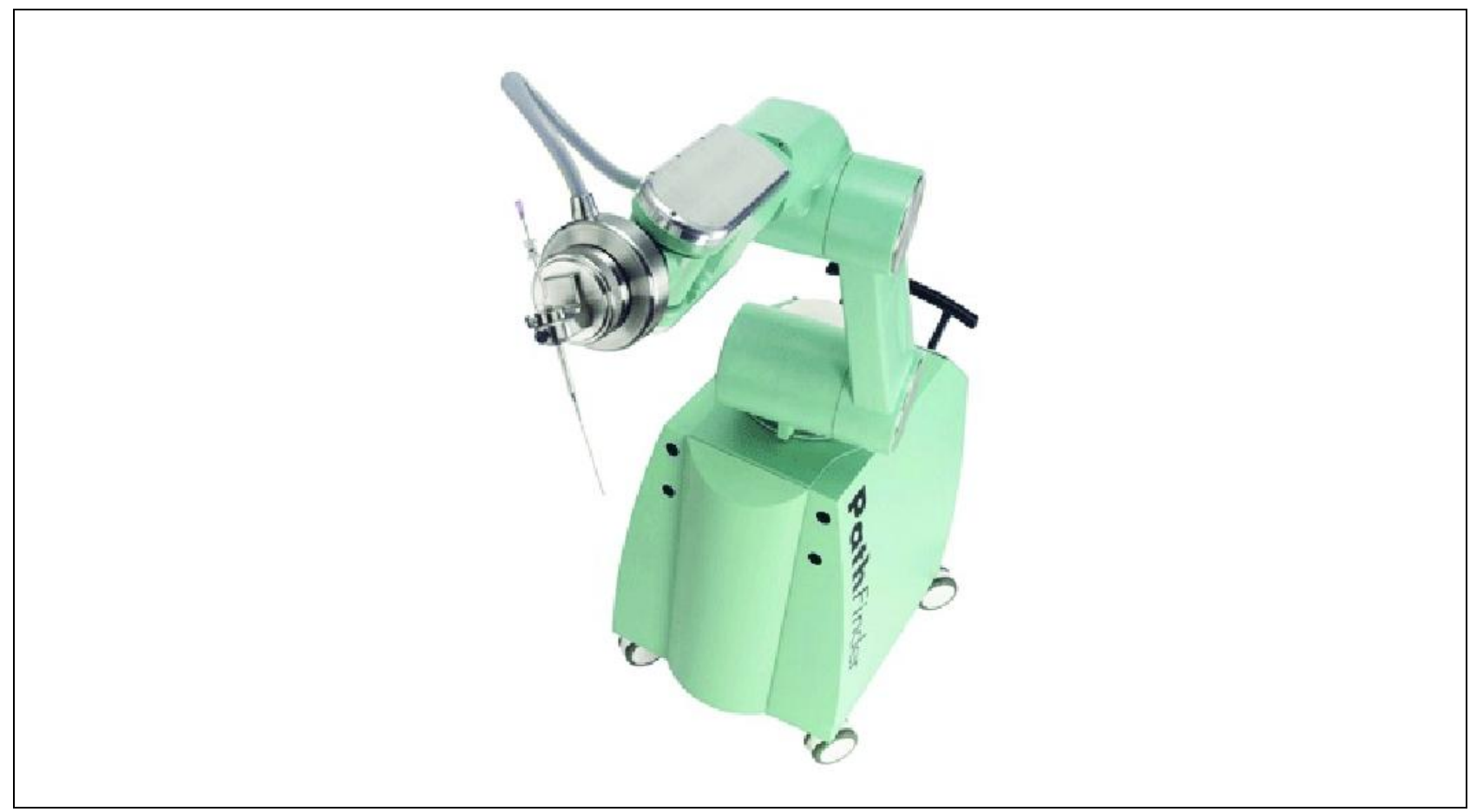

Fig.7. Prosurgics Pathfinder (Anonymous 4, 2012)

\subsection{Advantages and Disadvantages of Robotic Surgery}

\subsubsection{Advantages}

Robotic surgical systems have several potential advantages (Haidegger 2, 2008):

- superior 3D spatial accuracy provided by the robot,

- stabilization of the instruments within the surgical field,

- improvement of manual dexterity, motion scaling - very small controlled motions,

- tremor preventing,

- achieving minimally invasive procedures,

- integrated 3D vision system with high resolution,

- specific design for maximum performance, 
Nikolic, G.; Jerbic, B. \& Chudy, D.: Robotic Applications in Surgery with Special...

- advanced ergonomics,

- high-fidelity information integration,

- stable performance,

- invulnerability to environmental hazards,

- patient advantages (shorter recovery time),

- shorter hospitalization.

Advantages of the system are, as listed above, enormous and they overcome many previously reported deficiencies and obstacles in laparoscopic operations. Minimally invasive procedures, particularly minimum resection of brain tissue in neurosurgery, are achieved. Surgical robots are used for solving the most critical issues, such as:

- risk of damage on critical parts,

- limits of human dexterity,

- fatigue of the surgeon, who is not aware of it.

There are no significant objections to the existing teleoperating surgical robots, only high prices prevent them to be used more widely.

In 2003, experiments on humans (Johns Hopkins University) showed that an accuracy of $0.44 \pm 0.02 \mathrm{~mm}$ using a ROBOCAST robot was achieved, compared with an error of $0.98 \pm 0.02$ with a stereotactic frame and $1,96 \pm 1,6 \mathrm{~mm}$ with the standard (frameless) navigation system (Haidegger 1, 2008). It is not indicated whether these data apply only to the repetition of the same operation, or to the entire process of achieving the accuracy of the whole procedure. It can be assumed that these data do not apply to the complete work process, as the source data of the CT scan was performed with an interval of $0.7 \mathrm{~mm}$. Errors in the system are added and the total error is much higher.

\subsubsection{Disadvantages}

Technical solutions of existing neurosurgical robots do not provide more accurate positioning in relation to the existing procedures of stereotactic operations using stereotactic frames fixed to the patient's head which determines the threedimensional position of the operating point, i.e. of the target, based on the coordinates obtained from CT or MRI images. A usage of commercially available devices, circuits and parts due to the limitation of a total price for surgical robotic systems has, according to the principle of accumulation of errors, influence on the reduction of accuracy of the system. Likewise, for the approval of applications in the operating room a high degree of security and reliability is required, far higher than with other devices. This requires monitoring the movements of the robot, often with a triple safety system, which technically complicates the performance, but also increases the final price.

Most designed surgical robots has manipulators (arms) with a maximum of 4 to 6 degrees of freedom of motion, while the human wrist has 7 degrees of freedom. Also, as can be seen on the most frequently applied neurosurgical robots NeuroMata 
and ROSA, they have only one "arm." The surgeon's dexterity is achieved by synchronous movements and coordination with both arms together with extremely movable hands and fingers.

The previous surgical robots do not have two "arms" on account of higher prices, but also due to the problem of synchronization of both arms. Only ARTEMIS robots have two robotic arms that are controlled by the surgeon on the control console, but that robot is not designed for neurosurgery.

Apart from prices (which will decrease in the course of the technological development) and accuracy, another serious disadvantage of the system is the size of robots. They occupy a relatively large space, which is already limited in the operating room in which all devices must be in the patient's environment and available to surgeons and medical staff. An adequate storage space to accommodate the robotic system that is out of usage is also necessary. In autonomous robotic systems the problem of assessing the deviation of the position of the target points due to a shift of the brain through liquor leakage was observed. This problem of correction in the ongoing operation has not yet been solved using the robot itself and its sensory system. It is solved with other auxiliary devices which require additional space and restrict the application.

\section{The Application of a Dual-Arm Robotic System in Neurosurgery - RONNA (Jerbić et al. 2012, Jerbić et al. 2013, Nikolić et al. 2012, Stipančić et al. 2013)}

The above analysis showed that the existing neurosurgical robots have certain disadvantages arising from the concept of a robot that can be expressed as:

1. problem of absolute positioning accuracy due to the application of revolute classical kinematic architectures that cause the summation of errors both because of mechanical design and because of regulation,

2. action problem in anthropomorphic conditions and unstructured operating environments.

The research team of the Faculty of Mechanical Engineering and Naval Architecture (FSB), Zagreb (Croatia) in collaboration with the Clinical Hospital (KBD) of Zagreb and the Institute for Brain of Zagreb are developing a dual-arm robotic neurosurgical system that is currently at the stage of preclinical studies. The system is called RONNA - Robotic Neurosurgical Navigation and began to be developed in 2011 (Jerbić et al. 2013). These shortcomings of the previously developed neurosurgical robotic systems have been removed using a number of technical solutions for new technologies available on the market, from light-weight robots with 7 degrees of freedom, accurate position sensing system, forces and moments, laser and optical devices, as well as newly developed software packages. The basic idea is accurate reading of the coordinate system of the target point on the CT or MRI images in relation to the outer marker attached to the patient's head. Using the vision system, the robot finds the marker and connects it with the 
coordinates of the target point from the shots of the patient's head. Relevant operational programs determine the remaining part of the robot.

The RONNA robotic system is a specific, innovative approach to robotic applications in neurosurgery. It uses a developed new localization method to determine original coordinates on the patient's head, being the crucial problem of neurosurgical robotics. Two robot arms or independently controlled robots coordinate among themselves during localization, navigation and surgical procedures. In this way the disadvantages of robotic technology such as the accumulation of errors in positioning the open kinematic structure, are reduced, Figure 8. Localization of the patient's head is based on a special localization plate with markers attached to the patient's head.

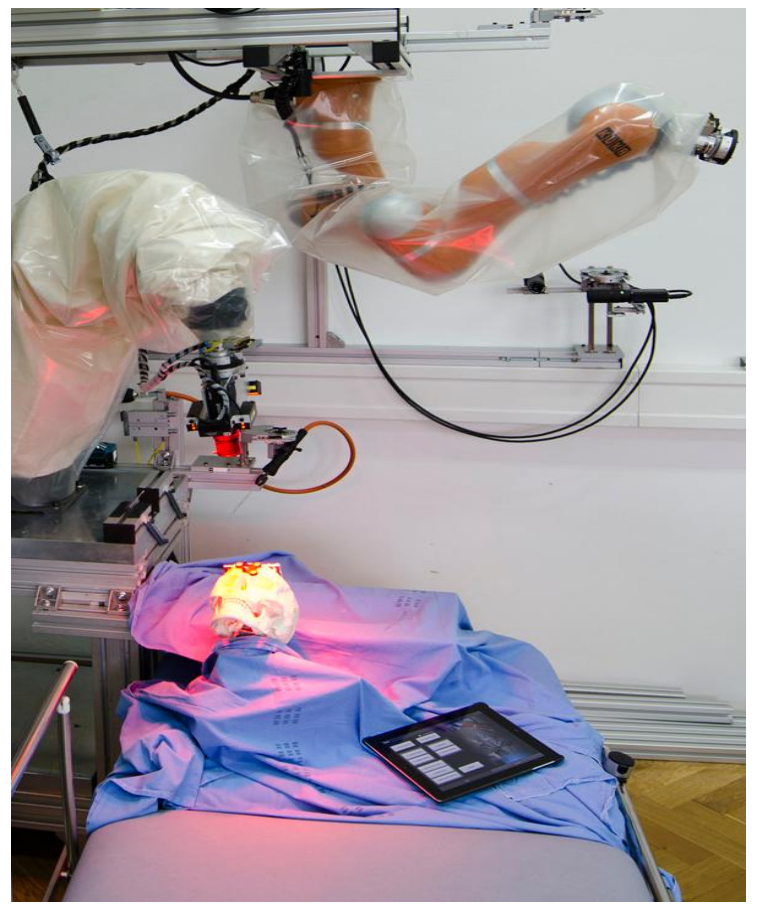

Fig. 8. RONNA robotic system in the Laboratory of the Faculty of Mechanical Engineering and Naval Architecture (Stipančić et al. 2013)

A positioning system located on the robotic arm consists of a camera and a laser distance meter, Figure 9. The starting coordinates of the robot are determined by finding the mounted localization plate with markers on the patient's head. The coordinates of the target are taken during planning for surgery from the shots made with CT or MRI. These coordinates are transmitted to the robot, and the link is the external mounted localization plate with markers, which determines the origin of the coordinate system. The robot gives the surgeon the ability to move the robot arm, but only in the direction and by the length that have been previously determined. If necessary, the robot can perform this operation by itself. The first option is the issue of security or ethical attitude about independent work of the robot. 


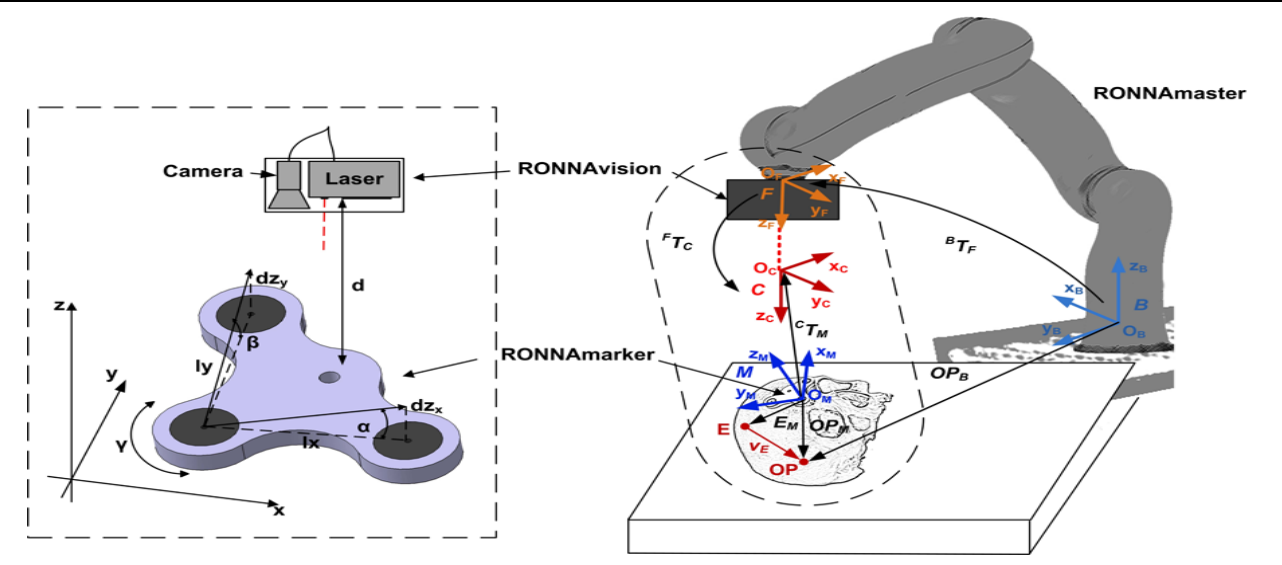

a)

b)

Fig. 9. Positioning system a) localization plate and vision system, b) spatial presentation of the RONNAvision and RONNAmarker system (Stipančić et al. 2013)

When planning for surgery, the spatial angle of entry of the surgical instrument is determined based on the developed program that takes into account the vital centres and blood vessels using a series of images that have been scanned in scanning step $(0.7 \mathrm{~mm})$.

The RONNA system consists of several modules that are connected to each other and work as a complete system. These include navigation and planning software RONNAplan, two robotic arms or two robots RONNAmaster with 6 degrees of freedom and RONNAassistant with 7 degrees of freedom, measurement device RONNAvision, RONNAmarker for localization, and calibration stereovision device RONNAstereo, surgical instruments and advanced software. The presentation emphasizes the fundamental principles of the developed system and describes in detail specifically developed scientific contributions in the field of intelligent control, contextual interpretation of the environment and intelligent interaction with people, both in terms of functionality and security.

\subsection{Robotic Arms or Robots}

A robot master (KUKA KR6) is a revolute robot with a reach of $900 \mathrm{~mm}$, load capacity of $6 \mathrm{~kg}$ and repeatability of $\pm 0.03 \mathrm{~mm}$. The robot is used as an alternative to the common stereotactic methods due to its rigidity. It is used for precise guidance of the robot slave or surgeon with surgical instruments according to the accurate work schedule. The other robot is a light-weight robot with 7 degrees of freedom (KUKA LWR 4), has a different architecture and is compatible with the previous one. The drives of the robot slave are equipped with torque sensors, which ensure a smooth interactive behaviour and adaptation to external resistance. The robot is programmed to prevent the possibility of unintended collision with the medical staff, patients or surrounding equipment.

The size and functional requirements for the application of robots in neurosurgery are much more demanding than those of conventional robotics, such as industrial applications. First of all, the overall size of the system must be such that it fits in the operating room and prevents interference with the work of the medical staff. On the other hand, the robotic system must be able to meet highly complex 
requirements in terms of spatial abilities, using the longest possible reach of the robotic arm. We analysed different options for the placement of the robot in the area surrounding the operating beds, separate placement in two locations, hung from the ceiling, etc. The obtained results indicate the possibility of using different configurations. The configuration with a robot-slave mounted on the ceiling (or hanging from the top of the holder) saves space, but requires a very stable and difficult basic construction. The currently selected option (not the final one) places neurosurgical robots in the RONNA system on a special table trolley with its accompanying control equipment, Figure 10.

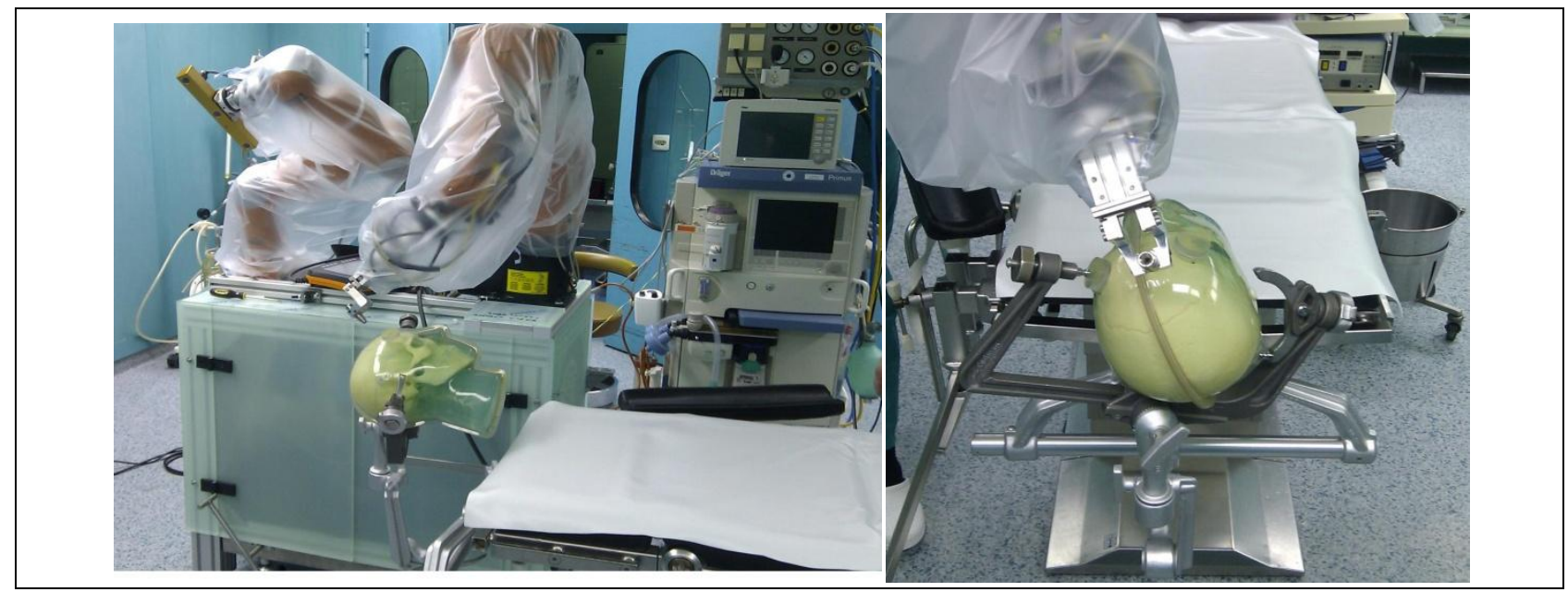

Fig. 10. RONNA in the operating room placed on the latest variant of the work platform

Since the RONNA configuration has two arms, a special control system based on an evolutionary algorithm that coordinates the movement of two robots, which prevents an unwanted collision, was designed. As the RONNA system is distributed and consists of two robots, a communication algorithm for the multi-agent robotic system developed in the earlier research at the Faculty of Mechanical Engineering and Naval Architecture is used.

\subsection{Markers - RONNA Marker}

The accuracy of the system is based largely on the module RONNAmarker that is a geometric interface between the robot localization procedure and CT or MRI. The localization plate is specific (Figure 9a). It is geometrically asymmetric and its features allow relatively precise registration. It consists of four parts, whose body is made of dark Duroplast with three cylindrical markers with blind holes in the middle, which are made of thermoplastic in bright colours and imprinted on the plate (Jerbic et al. 2012, Nikolic et al. 2012). These materials are used because they give a good contrast on CT and in the vision system. The localization plate with markers is attached to the patient's head and allows the localization of the patient. The marker has very narrow tolerances regarding surface flatness and precise position of cylindrical inserts. Three non collinear points are sufficient to describe the body in $3 \mathrm{D}$ space, creating a coordinate system. 
The localization plate with markers can be attached to the patient's head noninvasively and invasively. When a noninvasive method is used, the localization plate is glued using special glues. A suitable solvent is used to remove them from the skin. In case of an invasive method, a special screw (Jerbić et al. 2012) is used. The screw is made of titanium alloy with an appropriate structure that allows repetitive positioning of the localization plate and its fastening. Besides this important aspect of repeatability of assembly and disassembly while maintaining accuracy, it is extremely important to use only one screw, providing assured precision and reliability, which reduces the patient's trauma (Nikolic et al. 2012).

To verify the accuracy and repeatability of the localization plate with markers, a laboratory phantom was used for implementing the localization procedure. After making detailed measurements one can conclude the following: the measured accuracy of the repeatability process of the localization plate with markers has the same order of magnitude as the accuracy of robot repeatability. This suggests that the repeatability error of the localization plate is at least one order of magnitude lower than the accuracy of robot repeatability, which is acceptable. Maximum repositioning error is $0.24 \mathrm{~mm}$, while the overall mean error is $0.035+/-0.037 \mathrm{~mm}$. Hence, the positioning error is almost three times lower than in usual standard procedures in neurosurgical practice.

\subsection{Planning and Navigation - RONNA plan}

Based on MATLAB the software RONNAplan is designed for surgical planning and navigation. It has been developed to carry out the preparation of robotic surgery using CT or MRI scanned images. RONNAplan is able to interpret the DICOM standard format of tomographic scanned images and provide 2D and 3D visualization. The main purpose of the program is to define the surgical points in the patient's head $(\mathrm{x}, \mathrm{y}, \mathrm{z})$. The surgeon can determine the possible approximate path of motion (spatial orientation by angles $\alpha, \beta, \gamma$ ) of a surgical instrument in the operative field in the brain through the skull by guiding, connecting the selected points, Figure 11. The path is shown as a line that intersects all the associated scanned layers and the surgeon can check whether he passes through a delicate and dangerous area, and if necessary, modify the path.

During surgery the surgeon can monitor the progress of the movement of the surgical instrument on the scanned image of the brain using software RONNAplan.

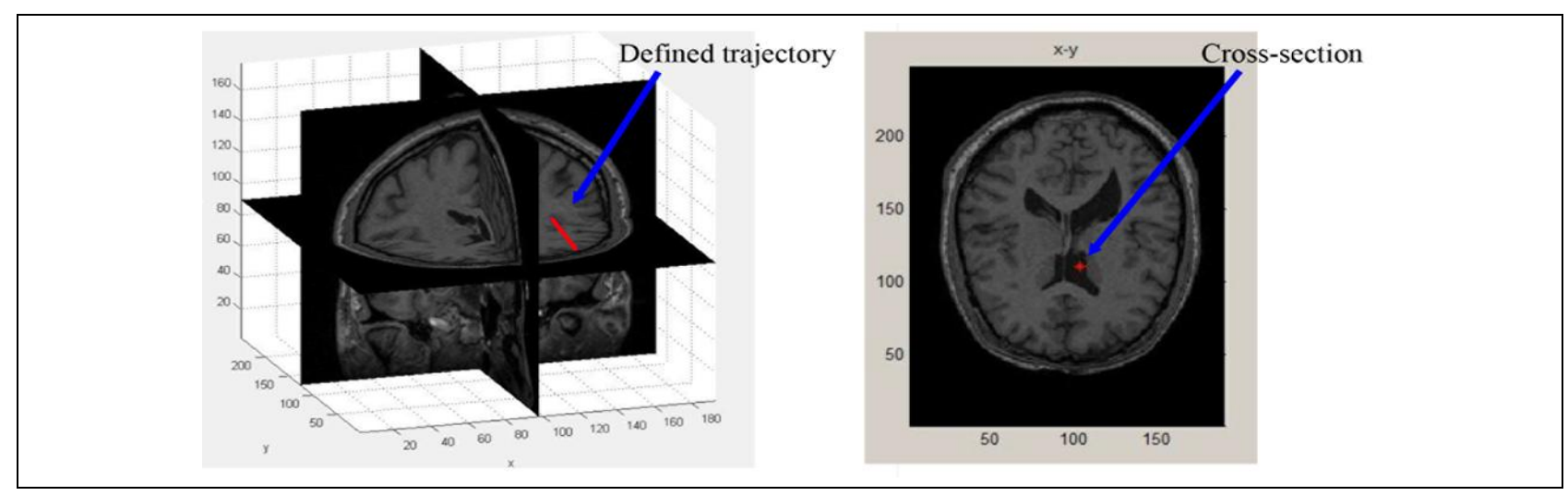

Fig. 11. Determining the path of the surgical instrument (Stipančić et al. 2013) 
Nikolic, G.; Jerbic, B. \& Chudy, D.: Robotic Applications in Surgery with Special...

\subsection{Localization device - RONNA vision}

RONNAvision performs the localization of the patient's head, using marks on the localization plate with markers attached to the patient's head. A prerequisite for the correct operation of the RONNA robot neurosurgical system is precise location. RONNAvision has a stereovision camera and a laser distance meter that are placed (interchangeable tool) on the robot master, Figure 12.

An audio-visual signal informs the surgeon which robot is in the proper position. The localization process after that is completely automated. The camera in the RONNA vision module is used to establish the exact position $(\mathrm{x}, \mathrm{y})$ and orientation $(\gamma)$ of the markers and is entered into the coordinates of the robot master. The robot master adjusts its position and orientation in accordance with the coordinate system of the markers. The other part of each step involves measurements. Multiple measurements minimize errors. Error and accuracy deviation of the markers on the localization plate $(\Delta \mathrm{x}, \Delta \mathrm{y}, \Delta \mathrm{z}, \Delta \alpha, \Delta \beta, \Delta \gamma)$ are determined after two consecutive localization cycles. After having achieved the maximum positioning quality, the RONNA system informs the surgeon to proceed with the work.

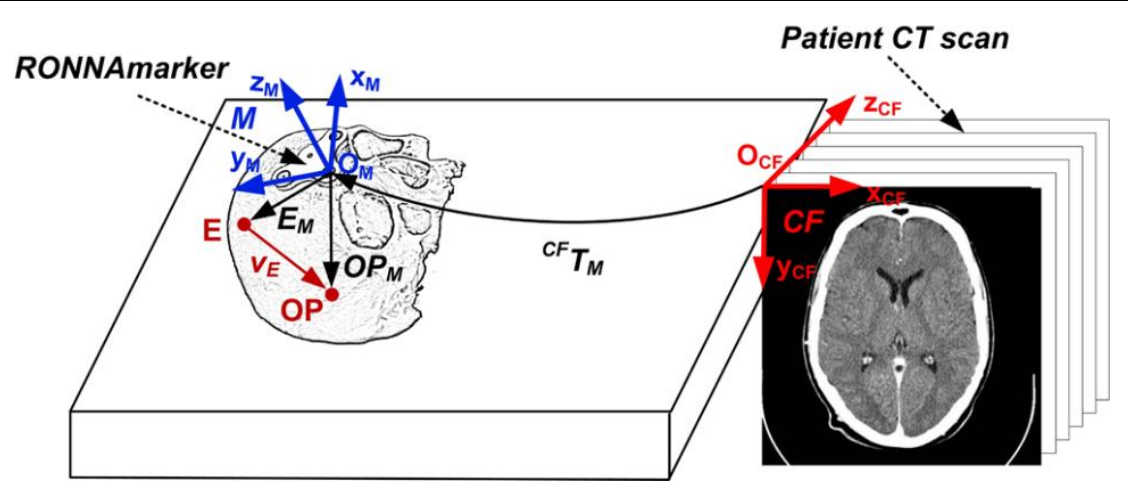

Fig. 12. Presentation of the connection between the two coordinate systems (Stipančić et al. 2013)

\subsection{Calibration of the Robot Master - RONNA stereo}

Despite the precise localization performed as described in the previous section, significant deviations are possible. They occur as a result of summation of errors in the open kinematic chain of the robot, inevitable inaccuracies in the design of tools / instruments and tool changers. When the robot (independently changing tools, instruments, and devices that are placed on the tool holder around the robot) replaces the vision system with a surgical medical instrument, it needs to be calibrated before surgery. This is done using a robot slave (stereovision module).

The robot master uses a special tool, a guide for directing surgical instruments such as needles, drills, or any other instrument to the planned position with the desired orientation. The guide also allows the surgeon to be in direct contact with the patient, without allowing the robot to take complete control of the surgical procedure (Figure 1 case a). The cameras are mounted at a right angle, and the focus point of both cameras is in the intersection of their optical axes. The robot slave uses a common coordinate frame with the robot master and is able to set up a centre system in relation to the centre line of the calibration pin. The stereovision system measures 
the distance from the centre of the ball to the calibration pin and brings the robot master into a relatively exact position. When the position of the calibration pin has been corrected, the original system data are updated according to the revised deviations. The application of the dual-arm working method is shown in Fig. 13. The robot master positions the guide to the correct position and spatial orientation. The robot slave inserts a surgical medical instrument through the guide at the beginning of surgery.

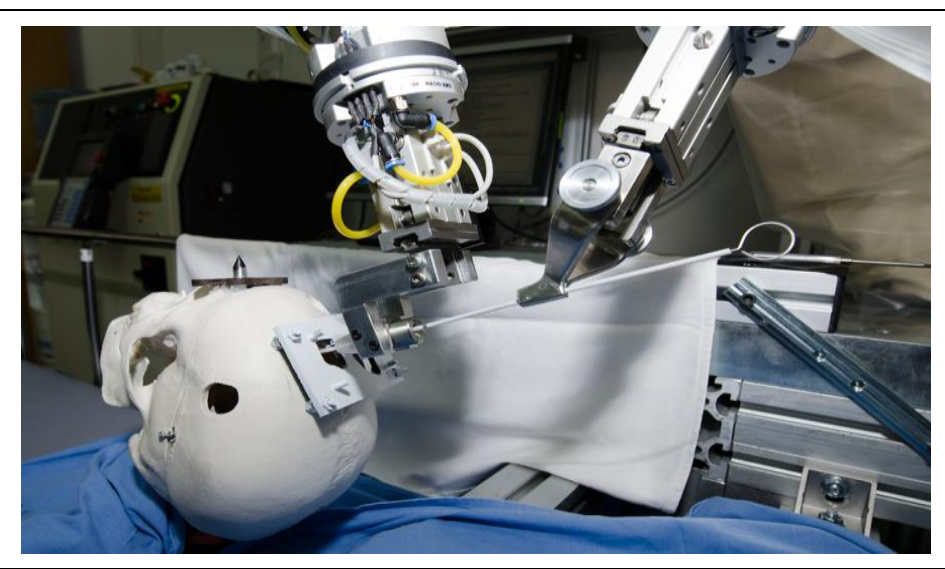

Fig. 13. The guide in the gripper of the robot master and catheter in the gripper of the robot slave (Stipančić et al. 2013)

The RONNA system includes all control functions based on sensors of power and torque in the impedance control incorporated in the mechanism of the robot slave KUKA LWR4, as well as advanced behavioural models. The application of robots in the human environment means the ability to work in unstructured environments and intuitive ability to interact with people. Work in an unstructured environment (operating room) means that the corresponding objects are not predefined in relation to the location and spatial orientation. Therefore, the robot cannot be programmed implicitly because the working conditions are not known, at least not for all of the factors (e.g. position and behaviour of the medical staff, place of other equipment). Behaviour and interaction of the robot with the environment must be based on the perception of the surrounding objects and the people who work with the robot. It must be spatial, in all three dimensions. Behaviour must be intuitive enabling the surgeon to deal with the technical aspects of the system application. In the RONNA system a dual stereovision system is used to allow a depth perception of the environment, not just the perception of object images in the scene. This clearly shows that the control of the RONNA is "intelligent" by adjusting the robot behaviour with respect to the current situation or surgical phases. Combining control algorithms with the principles of probability and contextual understanding, which are developed by us, RONNA is able to behave "smartly" bypassing obstacles, to move away from the medical staff, to prepare for expected actions on the basis of perception. The above described advanced control solutions contribute not only to a simple and reliable application of the system, but also increase safety, which is the inevitable issue in the application of such systems. 
Nikolic, G.; Jerbic, B. \& Chudy, D.: Robotic Applications in Surgery with Special...

\subsection{Results}

A series of experiments in laboratory conditions was conducted on the phantom. The phantom is a polymer replica of the human skull. At the top of the skull replica is attached a plate with measuring spikes. Spikes simulate certain areas in the brain. Two lines engraved on the spikes spaced by $1.5 \mathrm{~mm}$ are a reference scale for optical measurements of the vision system, Figure 14.

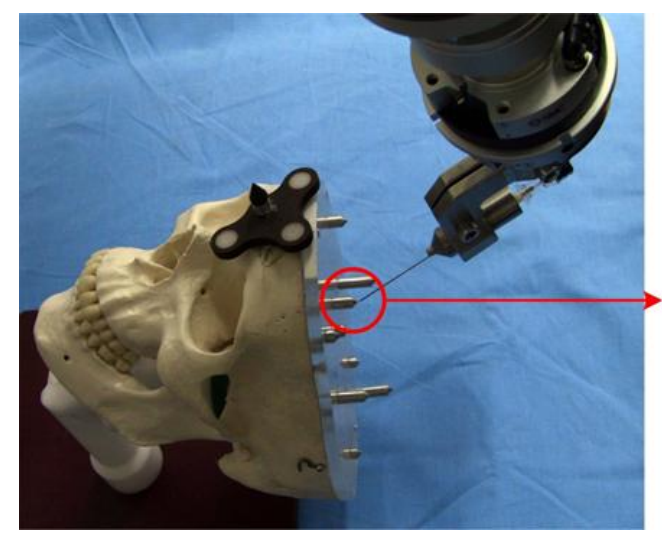

a)

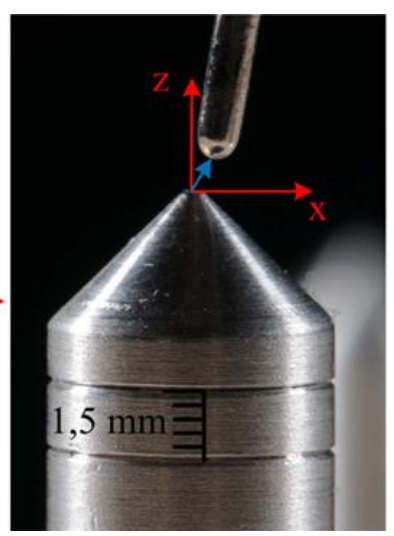

b)

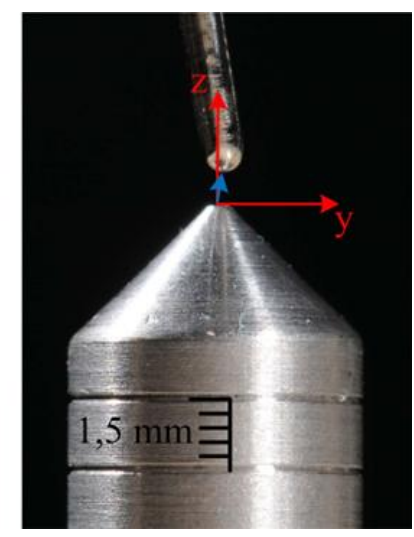

Fig. 14. Phantom a) skull with spikes, b) appearance of the spike and needle for checking the exact position (Stipančić et al. 2013)

RONNAmarker is attached to the head of the phantom to allow localization of the phantom, and so the registration of the initial coordinates of the robot. When the localization plate is attached to the forehead, the phantom is scanned by CT. The images are interpreted and the coordinates are determined in relation to the registered coordinate marker system. By using the vision system an error in the robotic system is determined. The tool placed at the end of the robotic arm - effector comes to the simulated surgical point (spike tip). Then the distance between the achieved position and the ideal position is measured. Using two vertical projections the vision system measures errors occurred in all three directions - $\mathrm{x} y$ and $\mathrm{z}$. Using precise reference engravings of the spikes, the image pixel values are converted into values in millimetres and subsequently the error distance $\mathrm{x} \mathrm{y}$ and $\mathrm{z}$ is calculated.

The repetition of the procedure with a phantom randomly mounted on the operating table provides a large sample of measurements from which one can calculate the accuracy of the system. The analysis of the results is divided into two parts - the area near the localization plate (distance less than $80 \mathrm{~mm}$ ), and the area farther from the plate (distance over $80 \mathrm{~mm}$, but less than $160 \mathrm{~mm}$ ). Also, the overall accuracy of the entire operative field is calculated. The analysis of results confirms the stability of the system performance and confirms the required accuracy. As expected, the precise results are better if the surgical procedure is performed closer to the localization plate, but even the surgical procedure at the farthest point stays within the desired tolerance. Figure 15 shows the location simulation of the RONNA system in the operating room. 


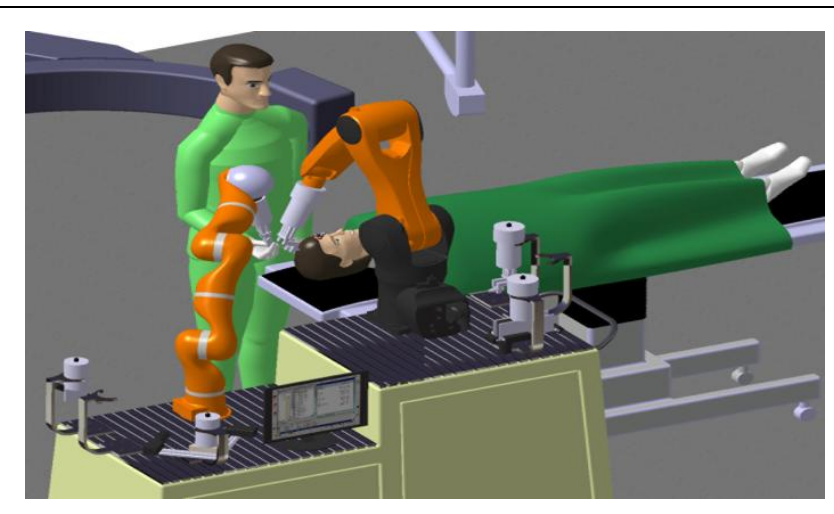

Fig. 15. Location simulation of one variant of the RONNA system in the operating room (Stipančić et al. 2013)

RONNA is a new application of robotics in neurosurgery using new methods of localization based on dual-arm configuration. This research focuses on the use of robots instead of the usual stereotactic method, thereby eliminating manual adjustment of devices and human error. Also, the applied robot of more complex configurations with two arms (or two robots) makes the system more flexible. The usage of robots is not only limited to the replacement of the application of stereotactic frames, but also to a number of other surgical procedures. Robots cooperate with each other so that a strenuous procedure, which is difficult to be performed by the surgeon, can be easily performed by the robot slave, in a simple, fast and more accurate way. After the preoperative phase, the RONNA system is capable of fully self-drilling, electrode installation and catheter insertion. The system has the potential for application in stereotactic encephalography (SEEG), biopsies, deep brain stimulation (DBS), resection of the tumour and the treatment of trigeminal neuralgia. Furthermore, dual-arm robotic configuration has certain advantages over the existing commercially available robot systems for neurosurgery. Operating mode with two arms ensures greater stability and rigidity of the kinematic chains of the robot. Kinematic structure of the dual-arm robot in the working process, such as drilling, inserting electrodes and catheter has only one degree of freedom, which provides better accuracy. Further research and development of the system will bring significant benefits in clinical practice, simplification and improvement of procedures for the surgeon and thereby benefit for the patient.

\section{Future Development of Robotics in Surgery}

Steps are shorter and shorter from science fiction to realization (Hockstein et al., 2007). It is inevitable that robotic surgery will develop faster and faster in several directions of development. It is expected that artificial intelligence will be increasingly present, voice communication, robots with more "arms" with sensors capable to detect forces and moments, also capable to recognize tissues, organs and their position in space. They will be also more accurate, capable to perform surgery procedures with minimum invasive treatment for the patients. Along with them not only new surgical instruments will be developed, but also a series of new procedures 
adapted to robot technology. Remote surgery using teleoperating robots will precede, but they will be replaced by fully autonomous robots (Lanfranco et al., 2004). In technical terms a great deal should be done to solve problems of this vision of application. The big problem remains the price what can last for a long time (Anonymous 7, 2012).

One of the development directions of robotics in medicine is miniaturization that can provide that classic surgery is not necessary any longer, but "surgery" and "treatment" would be performed within the patient's body (Nikolic, 2013).

Miniature robots are being developed; they can be swallowed like a pill and thus guided to the digestive system. They have "arms" or feelers with organic glue with which they can adhere to the intestinal wall; they can be stopped or guided to the spot to be examined additionally. Besides being able to send pictures of the interior for the purpose of diagnosis, taking samples using biopsy, can burn (cauterise) bleeding wounds using a laser, etc. Figure 16 (Carnegie Mellon University Nanorobotics Lab.).

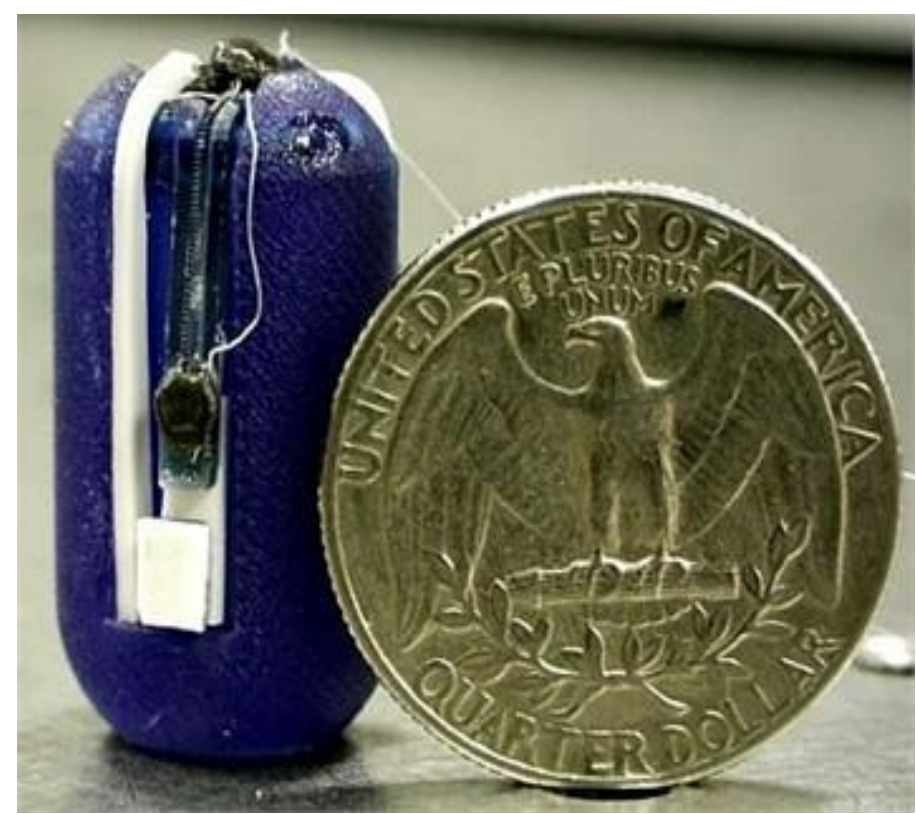

Fig. 16. Robotic pill for intestinal diagnosis (Uni. Carnegie-MelloN9

New micromachining technologies, integration of mechanics and electronics through the elements of MEMS or NEMS (Micro-Electro-Mechanical Systems, Nano Electro-Mechanical Systems) are responsible for creating miniature medical robots. Scientists around the world are working on creating the first usable medical miniature robot. At present, the development of miniature robots is in the early stage of a first prototype. Figure 17 shows the prototype of a miniature robot having the size of only $2 \mathrm{~cm}$ and weighing $5 \mathrm{~g}$. It was developed at Ritsumeikan University in Japan in 2007. Although it is an impressive achievement, in the near future nanorobots should be one thousand times smaller. 


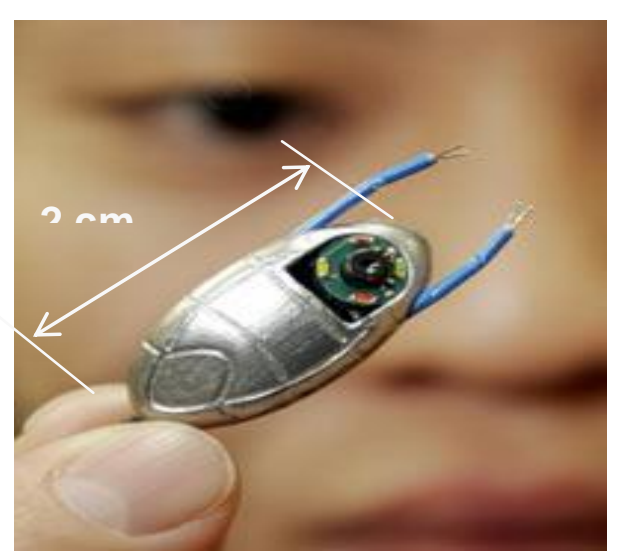

Fig. 17. Mini-robot (Ritsumeikan University, Japan)

Scientists of the Institute of Technology in Israel designed a micro-robot ViRob in 2009, measures only a few millimetres in length and only $1 \mathrm{~mm}$ in diameter, Figure 18 (Anon. 5, 2009). It uses small needles to crawl through blood vessels and its task is to administer medicine at a specific location in the body. Scientists have manipulated the magnetic field outside the body using the robot grippers. The magnetic field causes the vibration of robot needles (limbs), pushing it further through a blood vessel. For now, all the energy for the micro robot has come from an external source of energy. It is estimated that a relatively simple intervention in the construction can further reduce its size.

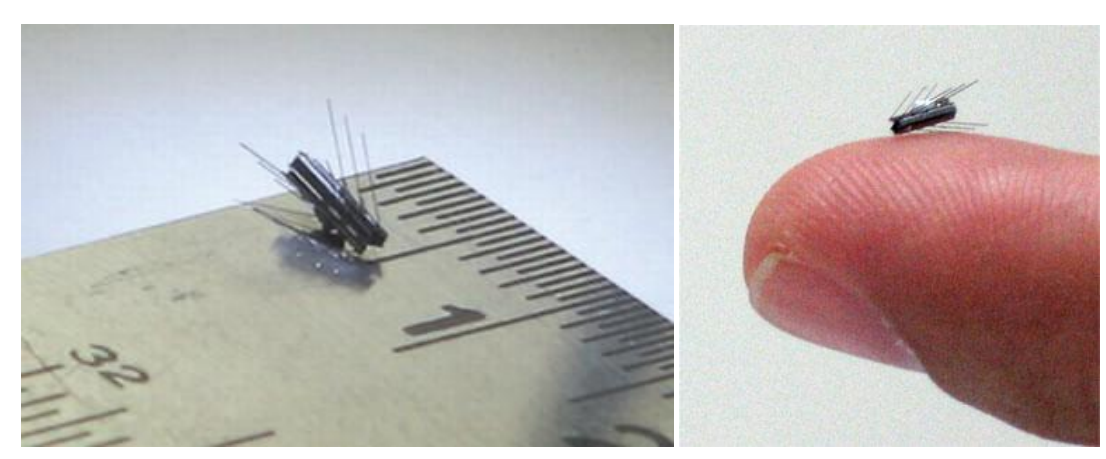

Fig. 18. Micro-robot with needles ViRob (Anonymous 5, 2009)

Further reducing the size of the micro-robot would enable its injection into the blood where it could move through blood vessels. It is believed that the following tasks could be performed:

- prevention and elimination of blood clots,

- destruction of cancer cells,

- removal of fatty plaques in the arteries (arteriosclerosis),

- removal of parasites,

- removal of bacteria and viruses,

- breaking up kidney stones,

- cleaning wounds and injuries by removing impurities from wounds, thereby reducing the possibility of infection, diagnostics, etc. 
Nikolic, G.; Jerbic, B. \& Chudy, D.: Robotic Applications in Surgery with Special...
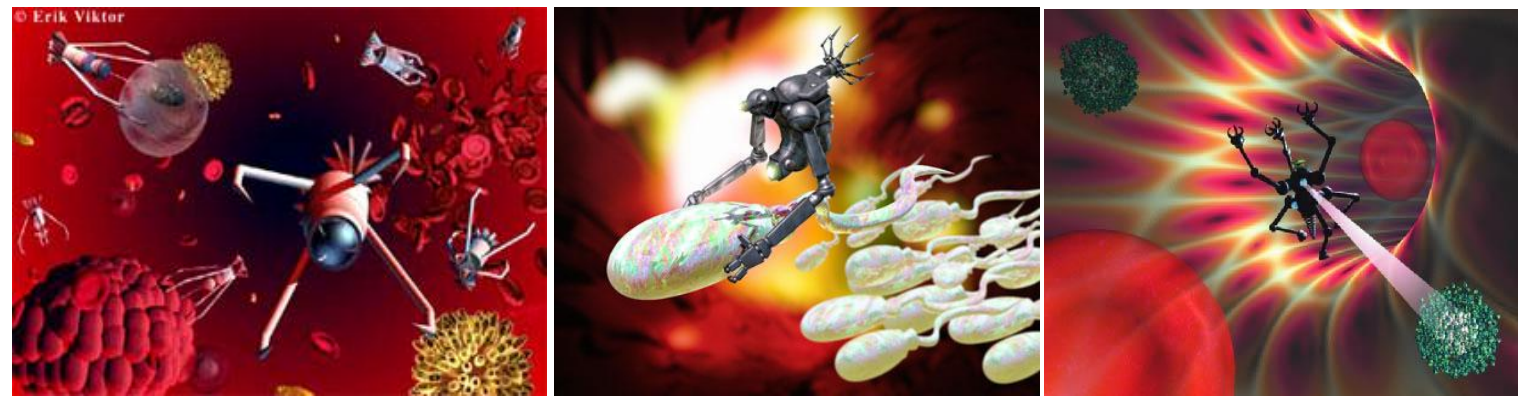

Fig. 19. Some design concepts of micro-robots

To solve problems of the implementation at this micro level, there is a need to resort to existing solutions in nature, by copying them or through certain modifications creating bionic nanorobots as an example shown in Figure 20, which has been created and is in the experimental stage.

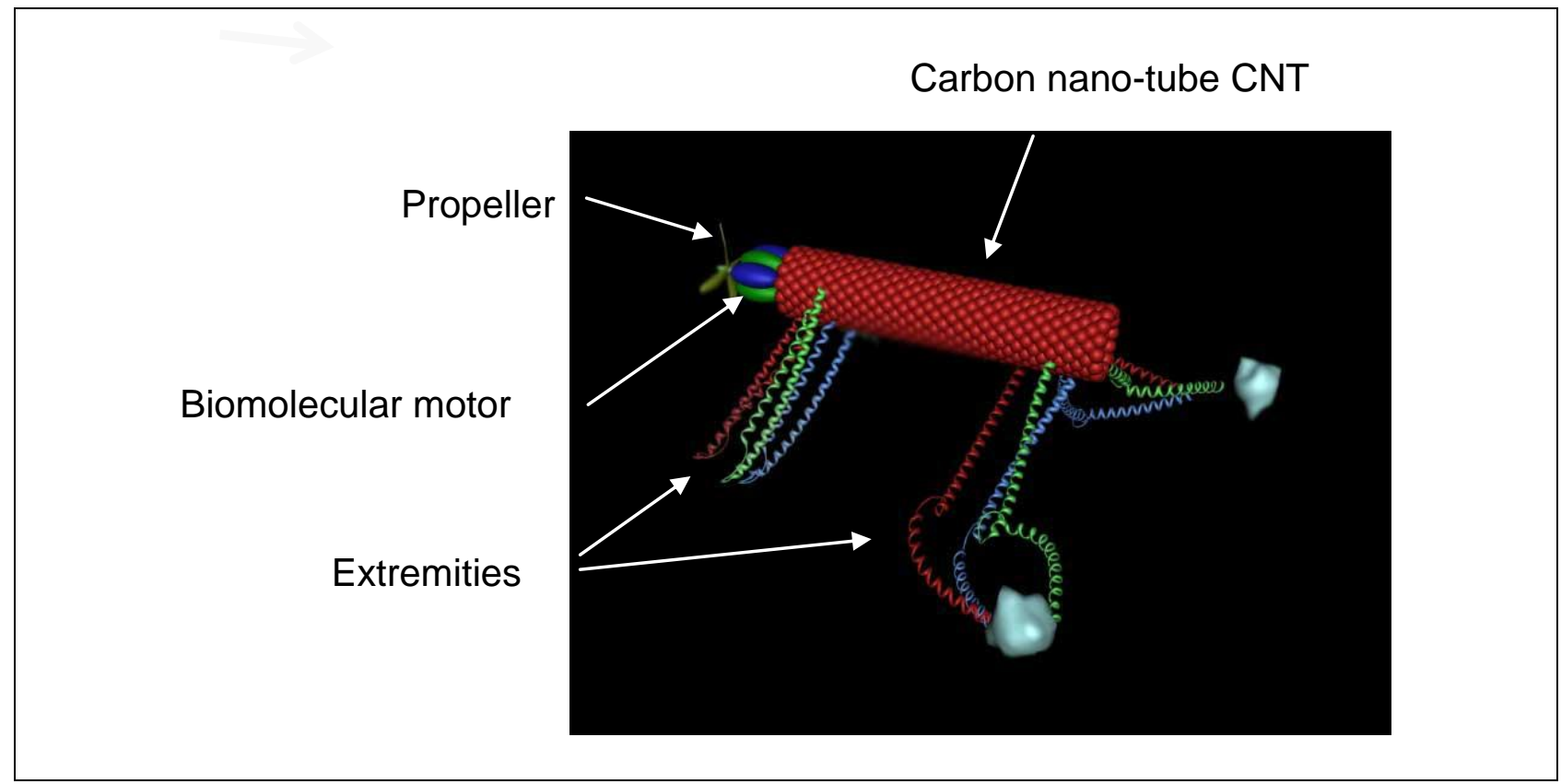

Fig. 20. Bionic nanorobot (Cornell University)

This vision of a bionic nanorobot called NANO-Copter is made of a carbon nano-tube, which forms the main body with flexible limbs that are used to move and manipulate objects, and a biomolecular motor located on the head with a propeller of $\mathrm{Ni}$. The bionic motor was created and worked for 40 minutes with $4 \mathrm{rpm}$ of the propeller. It was created from genetically modified Bacillus PS3 with E. coli (Cornell University in Ithaca, NY 2000).

Further considerations are aimed at the creation of a fully biological robot in the form of certain bacteria that have been genetically modified and perform useful tasks. Is it robotics or bionics, or is it only about biology? This is the reason why in this context all previous definitions of robotics are changed. New profiles of professionals who deal with these issues are bioinformaticians, bionic experts suggesting that only one area of specialization cannot solve such tasks even if it is their basic area of work. 


\section{Conclusion}

Surgical robotics is still in its infancy, but it has already proved to be of great importance, especially in areas inaccessible to conventional laparoscopic procedures. It remains to be seen whether robotic systems will replace conventional laparoscopic instruments for independent technically less demanding procedures in the future (Anonymous 8, 2012). Although the price in medicine does not have such an important influence, as it has in industry, expensive research and complex sophisticated devices can, however, play an important role in a given case.

We believe that the time of "independent" surgical systems that will be able to perform surgeries is yet to come and will make one of the biggest revolutions in surgery. It can be claimed that the RONNA system makes a contribution to these development trends, changing attitudes in medicine science and eliminating resistance to the introduction of independent surgical robots, which is likely to occur as soon as the practice shows that they make significantly fewer mistakes than surgeons.

Besides commercial effects arising from the development of the neurosurgical robot, there are other side benefits as building up the appropriate personnel, top educated, who can design the most complex devices, such as the applications of the mentioned robots. Besides, they are available for educating users (user training can cost up to $1 / 4$ of device costs) and also can take part in maintenance, where costs are also significant.

\section{References}

Advincula A. P. et al. (2007): Robots in Surgery, History, Current and Future Applications, Editor Faust R.A., Nova Science Publishers, Inc., New York, ISBN-13, 978-1-60021-386-1

Anonimus 1 (2013): Da Vinci Surgical system, [Internet], available on http://en.wikipedia.org/wiki/Da_Vinci_Surgical_System, [viewed on 2013-06-09]

Anonimus 10 (2005): Robotc Surgery - Neurosurgery, [Internet], available on http://biomed.brown.edu/Courses/BI108/BI108_2005_Groups/04/neurology.html [viewed on 2013-06-06]

Anonimus 2 (2004): History of Robotic Surgery, [Internet], available on http://biomed.brown.edu/Courses/BI108/BI108_2004_Groups/Group02/Group\%200 2\%20Website/history_robotic.htm, [viewed on 2013-06-10]

Anonimus 3 (2013): Innovative surgical technology, [Internet], available on www.medtechsurgical.com/Products/ROSA, [viewed on 2013-06-08]

Anonimus 4 (2012): Medical Robots: Curent Systems and Robots, [Internet], available on http://www.hindawi.com/journals/jr/2012/401613/figl/, [viewed on 2013-03-02]

Anonimus 5 (2009): Micro-robot ViRob, [Internet], available on http://fr.ubergizmo.com/2009/05/micro-robot-virob/, [viewed on 2011-12-06] Anonimus 6 (2012): Micro-surgery support system for neurosurgery, [Internet], available on http://www.houseofjapan.com/electronics/micro-surgery-supportsystem-for-neurosurgery [viewed on 2013-06-05] 
Nikolic, G.; Jerbic, B. \& Chudy, D.: Robotic Applications in Surgery with Special...

Anonimus 7 (2012): Roboti u kirurgiji su sigurniji i skuplji, agencija Reuters, [Internet], available on http://www.plivazdravlje.hr/vijesti/clanak/21776/Roboti-u-kirurgiji-susigurniji-i-skuplji.html [viewed on 2013-06-06]

Anonimus 8 (2012): Robotic Surgery, [Internet], available on http://www.cs.middlebury.edu/ rglaser/Final\%20Project.html, [viewed on 2013-06-10]

Anonimus 9 (2013): Robotska kirurgija,( Robotic Surgery), [Internet], available on http://hr.wikipedia.org/wiki/Robotska_kirurgija, [viewed on 2013-06-06]

Goto T. et al. (2003): Clinical application of robotic telemanipulation system in neurosurgery, J. Neurosurg 99, pp. 1082-1084,

Haidegger T. 1, (2008): Improving the Accuracy and Safety of a Robotic System for Neurosurgery, [Internet], available on Diploma_haidegger_neurosurg_robot.pdf, [viewed on 2013-03-02]

Haidegger T. 2, (2008): Theory and Method to Enhance Computer-Integrated Surgical Systems, [Internet], available on http://www.omikk.bme.hu/collections/phd/Villamosmernoki_es_Informatikai_Kar/201 1/Haidegger_Tamas/tezis_eng.pdf, [viewed on 2013-06-02]

Herron D. M. \& Marohn M. (2007): A consensus document on robotic surgery, SAGES-MIRA Robotic Surgery Consensus Group, Surgical Endoscopy, vol. 22, no. 2, pp. 313-325

Hockstein N. G. et al. (2007): A history of robots: from science fiction to surgical robotics, J Robotic Surg 1, pp. 113-118, DOI 10.1007/s11701-007-0021-2

Jerbić B. at al. (2013): RONNA - Robotic Neurosurgical Navigation, prepress article, Zagreb

Jerbić B. et al. (2012): Lokalizacijska pločica i set za njeno fiksiranje,( Localization plate and set its fixing) patent registration, br. P20121063A

Karli S. \& Ćustić M. (2007): Robotska revolucija u kirurgiji, ( The robotic revolution in surgery) Nacional br. 628

Kwoh Y. S. et al. (1988): A robot with improved absolute positioning accuracy for CT guided stereotactic brain surgery, IEEE Trans. Biomed. Engng, 35(2), pp. $153-161$

Lanfranco A. R. et al. (2004): Robotics surgery, Current perspective, Annals of Surgery

Nikolić G. (2013): Budućnost razvoja robota u području mikro i nano veličina,( The future development of robots in the field of micro and nano size,Scientific Symposium, Krivodol) prepress article, 2. Znanstveno stručno savjetovanje, Krivodol Nikolić G. et al. (2008): Roboti \& Primjena u industriji tekstila i obuće,( Robots \& Applications in the textile and footwear), Zrinski TTF, Zagreb, ISBN 978-953-710522-8

Nikolić G. et al. (2012): Lokalization plate for stereotactic surgery, EU patent, $\mathrm{DM} / 079$ 990, 22.12.2012

Stipančić T. et al. (2013): Robotic Neurosurgical Navigation, [Internet], available on www.ronna.fsb.hr, [viewed on 2013-06-02] 